\title{
Activities of maturation-promoting factor (MPF) and mitogen-activated protein kinase (MAPK) are not required for the global histone deacetylation observed after germinal vesicle breakdown (GVBD) in porcine oocytes
}

\author{
Tsutomu Endo, Kunihiko Naito, Sachi Kume, Yukio Nishimura, Koji Kashima and Hideaki Tojo \\ Laboratory of Applied Genetics, Graduate School of Agriculture and Life Sciences, University of Tokyo, Bunkyo-ku, \\ Tokyo 113-8657, Japan \\ Correspondence should be addressed to K Naito; Email: aknaito@mail.ecc.u-tokyo.ac.jp
}

\begin{abstract}
The acetylation of nuclear core histone has been suggested to work as an epigenetic mark for transmitting gene expression patterns to daughter cells. Global histone deacetylations, presumably involved in the reprogramming of the gene expression, have been observed after germinal vesicle breakdown (GVBD) in a cell cycle-dependent manner during meiotic maturation of mouse and porcine oocytes, although the regulation mechanism of histone deacetylation has not been studied well. In the present study, we examined the involvement of a crucial cell-cycle-regulator, maturation-promoting factor (MPF), and a meiosis-related kinase, mitogen-activated protein kinase (MAPK), in the global histone deacetylation during porcine oocyte maturation. In order to know whether the activities of MPF and MAPK were required, or the breakdown of GV membrane was sufficient, for the global histone deacetylation observed after GVBD, we artificially destroyed the GV membrane of the porcine immature oocytes. The artificial GV destruction (AGVD) induced histone deacetylation without the activation of MPF and MAPK. This deacetylation after AGVD was not affected by an MPF inhibitor, roscovitine, or an inhibitor of protein synthesis, cycloheximide, but was completely prevented by an inhibitor of histone deactylases (HDACs), trichostatine A. HDAC1 was present in the GV of the immature oocytes and localized on chromosomes after GVBD and AGVD. These results suggest that the MPF and MAPK activities were dispensable and the breakdown of the GV membrane was sufficient for the global histone deacetylation, which was catalyzed by HDAC activity

Reproduction (2006) 131 439-447
\end{abstract}

\section{Introduction}

Nuclear core histones undergo various covalent modifications, such as phosphorylation, methylation and acetylation (Wu \& Grunstein 2000). These post-transcriptional modifications not only influence chromosome structures and functions (Spencer \& Davie 1998, Wu \& Grunstein 2000), but also have been suggested to play a role as epigenetic marks (Strahl \& Allis 2000, Turner 2002). Histone $\mathrm{H} 3(\mathrm{H} 3)$ and histone $\mathrm{H} 4(\mathrm{H} 4)$ can be acetylated at each of four lysine residues on their $\mathrm{N}$-terminal tails: the lysines at positions 9 (K9), 14 (K14), 18 (K18) and 23 (K23) for $\mathrm{H} 3$, and the lysines at positions 5 (K5), 8 (K8), 12 (K12) and 16 (K16) for H4 (O'Neill \& Turner 1995, Agalioti et al. 2002). In somatic cells, acetylations on the lysines far from the $\mathrm{N}$-terminal end, $\mathrm{K} 14$ of $\mathrm{H} 3$, and $\mathrm{K} 12$ and $\mathrm{K} 16$ of $\mathrm{H} 4$, are maintained even in the M-phase, and therefore these acetylations have been indicated to work as a type of cell memory that transmits gene expression patterns to daughter cells (Kruhlak et al. 2001, Kanno et al. 2004). On the other hand, a global histone deacetylation was observed during meiotic maturation of mouse oocytes, and was suggested to be associated with the reprogramming of the gene expression. (Kim et al. 2003, Sarmento et al. 2004). In spite of the probable importance of histone acetylation in the genome epigenesis, the regulation mechanism of this histone modification during oocyte maturation has not been studied except for only one report (Akiyama et al. 2004).

We recently analyzed the histone acetylation states of porcine oocytes throughout the meiotic maturation by immunocytochemical methods (Endo et al. 2005). In the report, we showed that all of the lysines examined were highly acetylated during the germinal vesicle (GV) stage, and then deacetylated by histone deacetylases (HDACs) after germinal vesicle breakdown (GVBD) at the first and 
the second metaphases with a transient reacetylation at the first anaphase and telophase. We have suggested the high cell-cycle dependency of the histone deacetylation during porcine oocyte meiosis, as this fluctuation pattern of histone acetylation showed a strong inverse correlation with the activity level of a crucial cell-cycle regulator, maturation-promoting factor (MPF), reported in porcine oocytes (Naito \& Toyoda 1991). Since MPF activates just before GVBD and phosphorylates many substrates to induce meiotic events, it is conceivable that MPF regulates the activities of HDACs, which are regulated by phosphorylation (Pflum et al. 2001, Galasinski et al. 2002, Tsai \& Seto 2002). In addition to MPF, it has been well established that the mitogen-activated protein kinase (MAPK) is also activated around GVBD and involved in the regulation of meiotic progression (Inoue et al. 1995, 1996). Because the pronounced histone deacetylation occurred $6 \mathrm{~h}$ after GVBD, when MPF and MAPK were activated, the involvement of these meiosis-related kinases in the regulation of the global histone deacetylation during meiosis was highly expected. On the other hand, as the GVBD allows the interaction of GV materials with cytoplasmic factors, it is also possible that the deacetylation depends on GVBD itself. In mouse oocytes, MPF has been suggested to play a role in the meiotic histone deacetylation, because the inhibition of MPF activity by an MPF-specific inhibitor, roscovitine, was shown to result in the disappearance of histone deacetylation (Akiyama et al. 2004). In this previous report, however, the authors could not exclude the latter possibility, since the inhibition of MPF activity was always associated with the inhibition of GVBD. At present, there has been no other report on the regulation of histone deacetylation during meiotic maturation of oocytes.

In the present study, we attempted to determine whether the activities of meiosis-related kinases were required, or the breakdown of GV membrane was sufficient, for the global histone deacetylation observed after GVBD. Therefore, we artificially destroyed the GV membrane of the porcine immature oocytes and then observed the status of histone acetylation. The intracellular localization of HDAC1 was also examined immunocytochemically, and the regulation of meiotic histone acetylation was discussed.

\section{Materials and Methods}

\section{Collection and maturation culture of porcine oocytes}

Porcine oocytes were obtained as described previously (Endo et al. 2005). Briefly, porcine cumulus-oocyte complexes (COCs) were aspirated from follicles $(2-5 \mathrm{~mm}$ in diameter) of gilt ovaries obtained from a slaughterhouse. Groups of 20-25 COCs with intact, unexpanded cumulus cells were cultured in drops of $0.1 \mathrm{ml}$ culture medium for up to $48 \mathrm{~h}$ at $37^{\circ} \mathrm{C}, 100 \%$ humidity and $5 \% \mathrm{CO}_{2}$ in air. The culture medium consisted of modified Krebs-Ringer bicarbonate solution (TYH) (Toyoda et al. 1971), $1.0 \mathrm{IU} / \mathrm{ml}$ pregnant mare's serum gonadotropin (Sankyo, Tokyo, Japan), $3.2 \mathrm{mg} / \mathrm{ml}$ BSA (Sigma) and 20\% porcine follicular fluid collected as described previously (Naito et al. 1988). For treatment with inhibitors, $500 \mathrm{nM}$ trichostatin A (TSA; Wako Pure Chemical, Osaka, Japan), $50 \mu \mathrm{M}$ roscovitine (Sigma) or $35 \mu \mathrm{M}$ cycloheximide (Sigma) were added to the culture medium.

\section{Artificial GV destruction (AGVD)}

Before micromanipulation, the oocytes were denuded with $150 \mathrm{IU} / \mathrm{ml}$ hyaluronidase (type IV, Sigma) and gentle pipetting. The denuded oocytes were centrifuged at 15000 r.p.m. for $5 \mathrm{~min}$ at room temperature to localize cytoplasmic lipid droplets and visualize the GV (Fig. 1A and $\mathrm{D})$. The centrifuged oocytes were incubated in the culture medium supplemented with $15 \mu \mathrm{g} / \mathrm{ml}$ cytochalasin B, $10 \mathrm{mg} / \mathrm{ml}$ sucrose and $10 \mu \mathrm{g} / \mathrm{ml}$ Hoechst 33342 for $10 \mathrm{~min}$ at $37^{\circ} \mathrm{C}$. The GV was aspirated with a beveled suction pipette (outer diameter, $10-15 \mu \mathrm{m}$ ) attached to a micromanipulator (Narishige, Tokyo, Japan) in order to break the GV membrane and suck out GV contents
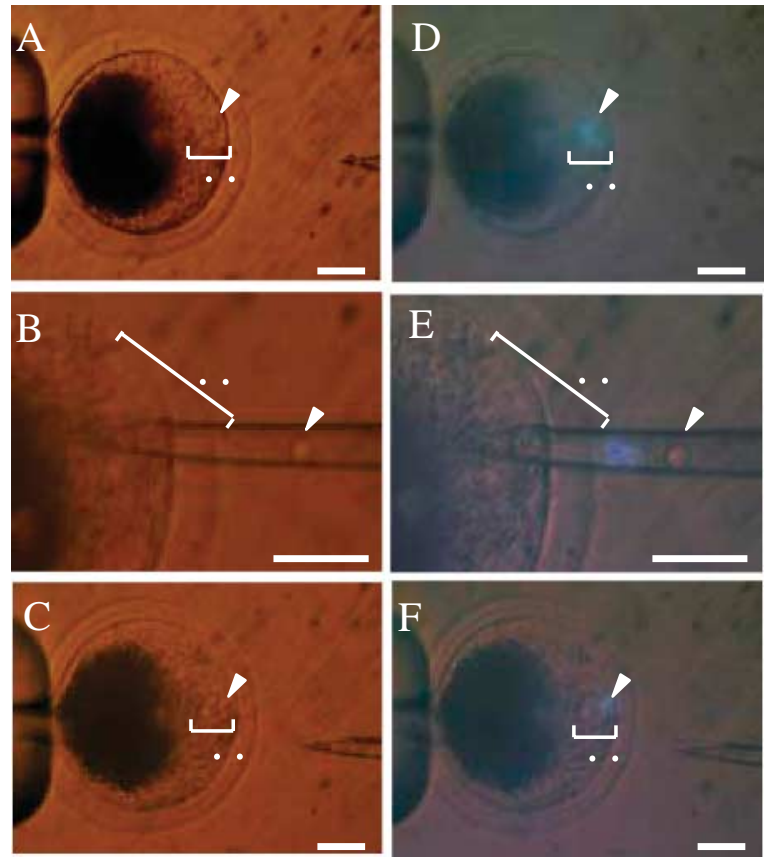

Figure 1 Methods used for artificial GV destruction (AGVD). Bright fields $(A-C)$ and the merged images of the bright fields and the DNA staining with Hoechst $33342(\mathrm{D}-\mathrm{F})$ are shown. (A and D) A porcine oocyte centrifuged at 15000 r.p.m. for 5 min to localize cytoplasmic lipid granules and visualize the GV. The solid star and arrowhead indicate the GV and nucleolus respectively. (B and E) The GV was aspirated with a beveled suction pipette in order to break the GV membrane and suck out the GV contents. The solid star indicates the range of the GV membrane. The nucleolus (arrowhead) is outside the GV membrane. ( $\mathrm{C}$ and F) The GV contents were placed back within the cytoplasm. The solid star indicates the GV materials containing a nucleolus (arrowhead). Scale bar: $30 \mu \mathrm{m}$. 
(Fig. 1B and E). Thereafter, the GV contents were placed back within the cytoplasm (Fig. 1C and F). These oocytes were referred to as AGVD oocytes. The AGVD oocytes were cultured as described above, and then some of them were subjected to an examination of nuclear status by phase-contrast microscopy after fixation with acetic acidethanol (1:3) and staining with $0.75 \%$ aceto-orcein solution.

\section{Immunocytochemistry}

Fixation, permeabilization and blocking of the denuded oocytes were performed as described previously (Endo et al. 2005). Then, the oocytes were treated with anti-K12 acetylated $\mathrm{H} 4$ antibody (06-761, Upstate Biotechnology, Lake Placid, NY, USA), anti-K9 acetylated H3 antibody (9671S, Cell Signaling Technology, Beverly, MA, USA), anti-K9 tri-methylated $\mathrm{H} 3$ antibody $(07-212$, AbCam Ltd, Cambridge, UK), or anti-HDAC1 antibody (sc-6298; Santa Cruz Biotechnology, Santa Cruz, CA, USA) overnight. After washing, the oocytes were incubated in the mixture of fluorescein isothiocyanate (FITC)-conjugated antirabbit IgG (1:100 dilution with PBS; DAKO, Glostrup, Denmark) or antigoat IgG (1:400 dilution with PBS; Sigma) for 30 min. The oocytes were counterstained with Hoechst 33342 to visualize the chromosomes, and then examined under a confocal laser scanning microscope (LSM510-V2.01, Axioplan MOT; Carl Zeiss, Oberkochen, Germany).

\section{Immunoblotting}

The micro-Western blotting method (Naito et al. 1999) was used with several modifications. In all cases, 10 denuded oocytes were put in $2 \mu \mathrm{l}$ saline supplemented with $0.1 \%$ PVP, added to $0.5 \mu \mathrm{I} 5 \times$ Laemmli (1970) buffer, and denatured at $100{ }^{\circ} \mathrm{C}$ for $5 \mathrm{~min}$. Proteins were separated on a modified $10 \%$ polyacrylamide gel (Inoue et al. 1995) by SDS-PAGE and transferred to a polyvinylidene difluoride membrane (Immobilon Transfer Membranes; Millipore, Billerica, MA, USA). After blocking the membrane with $5 \%$ milk for $1 \mathrm{~h}$, the membrane was treated with anti-cyclin B1 monoclonal antibody (05-158; Upstate Biotechnology), anti-cyclin B2 polyclonal antibody (N-20; Santa Cruz Biotechnology), anti-CDK1 monoclonal antibody (sc-54; Santa Cruz Biotechnology) or antiMAPK polyclonal antibody (K-23; Santa Cruz Biotechnology, Santa Cruz, CA). Signals were detected by a blotting detection kit (Amersham Pharmacia Biotech) according to the manufacturer's instructions.

\section{MPF and MAPK activity assay}

Ten denuded oocytes were lysed in $2.5 \mu \mathrm{l}$ assay buffer (Naito \& Toyoda 1991) and stored at $-80^{\circ} \mathrm{C}$ until use. The activities of MPF and MAPK were evaluated in terms of the histone $\mathrm{H} 1$ kinase and myelin basic protein (MBP) kinase activities respectively, as described in previous reports (Sugiura et al. 2001, Kuroda et al. 2004).
The lysates $(2.5 \mu \mathrm{l})$ were added to $2.5 \mu \mathrm{l}$ of $2.5 \mu \mathrm{M}$ cAMPdependent protein kinase inhibitor (Sigma), $5 \mu \mathrm{l}$ of $2 \mathrm{mg} / \mathrm{ml}$ concentration of histone $\mathrm{H} 1$ (Sigma), $2.5 \mu \mathrm{l}$ of $10 \mathrm{mg} / \mathrm{ml}$ concentration of MBP (Sigma), and $5 \mu \mathrm{l}$ of $0.1 \mathrm{mM}\left[\gamma_{-}{ }^{32} \mathrm{P}\right]$ ATP $(0.4 \mathrm{mCi} / \mathrm{ml}$; Amersham Pharmacia Biotech), and the reaction was performed at $37^{\circ} \mathrm{C}$ for $1 \mathrm{~h}$. After the reaction, $5 \mu \mathrm{l}$ of $5 \times$ Laemmli buffer were added to each lysate, which was then denatured at $100^{\circ} \mathrm{C}$ for $5 \mathrm{~min}$ and subjected to SDS-PAGE. The bands of phosphorylated histone $\mathrm{H} 1$ and MBP were visualized after autoradiography.

\section{Results}

\section{Chromatin status of AGVD oocytes cultured in vitro}

In the intact, noncultured oocytes, the chromatin was localized at the central portion of the GV and surrounded the nucleolus (Figs $1 \mathrm{~A}$ and $\mathrm{D}$, and $2 \mathrm{~A}$ ). The chromatin was easily separated from the nucleolus when the GV membrane was broken and the GV contents were sucked into the pipette (Fig. 1B and E). After the contents were returned to the ooplasm, the chromatin aggregated near the nucleolus but did not surround it, and the shape of the chromatin was different from that in intact oocytes (Figs $1 \mathrm{C}$ and $\mathrm{F}$, and $2 \mathrm{~B})$. In order to examine whether the heteromorphic chromatin in the AGVD oocytes could complete meiotic maturation, the oocytes were cultured up to $48 \mathrm{~h}$. After 24-h culture, the chromatin clustered like a prometaphase chromosome (Fig. 2C), and almost no degenerative changes were observed in the AGVD oocytes morphologically. However, about half of the AGVD
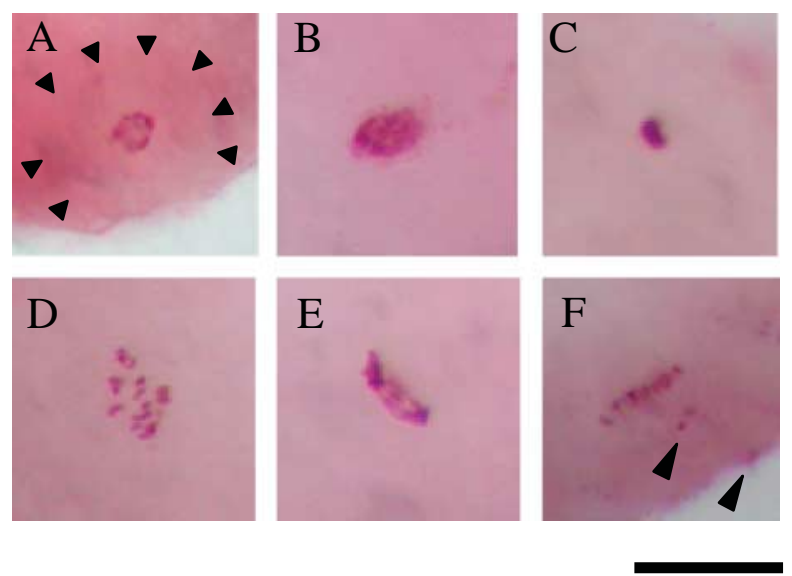

Figure 2 In vitro maturation of AGVD oocytes. (A) Intact noncultured oocytes at the GV stage. Arrowheads indicate the GV membrane. (B) An AGVD oocyte immediately after the manipulation and without cultivation. (C) An AGVD oocyte cultured for $24 \mathrm{~h}$. Chromosomes were clustered as in the prometaphase stage. (D) A 30-h-cultured AGVD oocyte at the first metaphase. (E) A 30-h-cultured AGVD oocyte at the first anaphase. (F) A 48-h-cultured AGVD oocyte at the second metaphase. Arrowheads indicate parts of the first polar body. The oocytes were stained with $0.75 \%$ aceto-orcein solution. Scale bar: $30 \mu \mathrm{m}$. 
oocytes stopped meiosis at this stage, and the remaining oocytes formed a first metaphase-like plate at $30 \mathrm{~h}$ of culture (Fig. 2D). Then the oocytes emitted their first polar bodies (Fig. 2E), and about 30\% of the AGVD oocytes reached the second metaphase (MII) at $48 \mathrm{~h}$ of culture (Fig. 2F). This result indicates that at least some of the AGVD oocytes could complete meiotic maturation, although the AGVD manipulation had some deteriorative effects on the oocyte meiosis, as the maturation rate of intact oocytes was more than $80 \%$ in our culture system (data not shown).

\section{Changes of histone modifications in AGVD oocytes}

Examples of the immunostaining of histone modifications in AGVD oocytes are shown in Fig. 3. As reported previously (Endo et al. 2005), the degrees of histone acetylations were high at the GV stage in both $\mathrm{K} 9$ on $\mathrm{H} 3$ (Fig. 3A) and $\mathrm{K} 12$ on $\mathrm{H} 4$ (Fig. 3E), representing the lysines near the $\mathrm{N}$-terminal end and far from the $\mathrm{N}$-terminal end respectively. The degrees of histone acetylation just after manipulation ( $0 \mathrm{~h}$ : Fig. 3B and F) were almost the same as those in intact GV oocytes, indicating that AGVD manipulation itself had no effect on the histone acetylation.

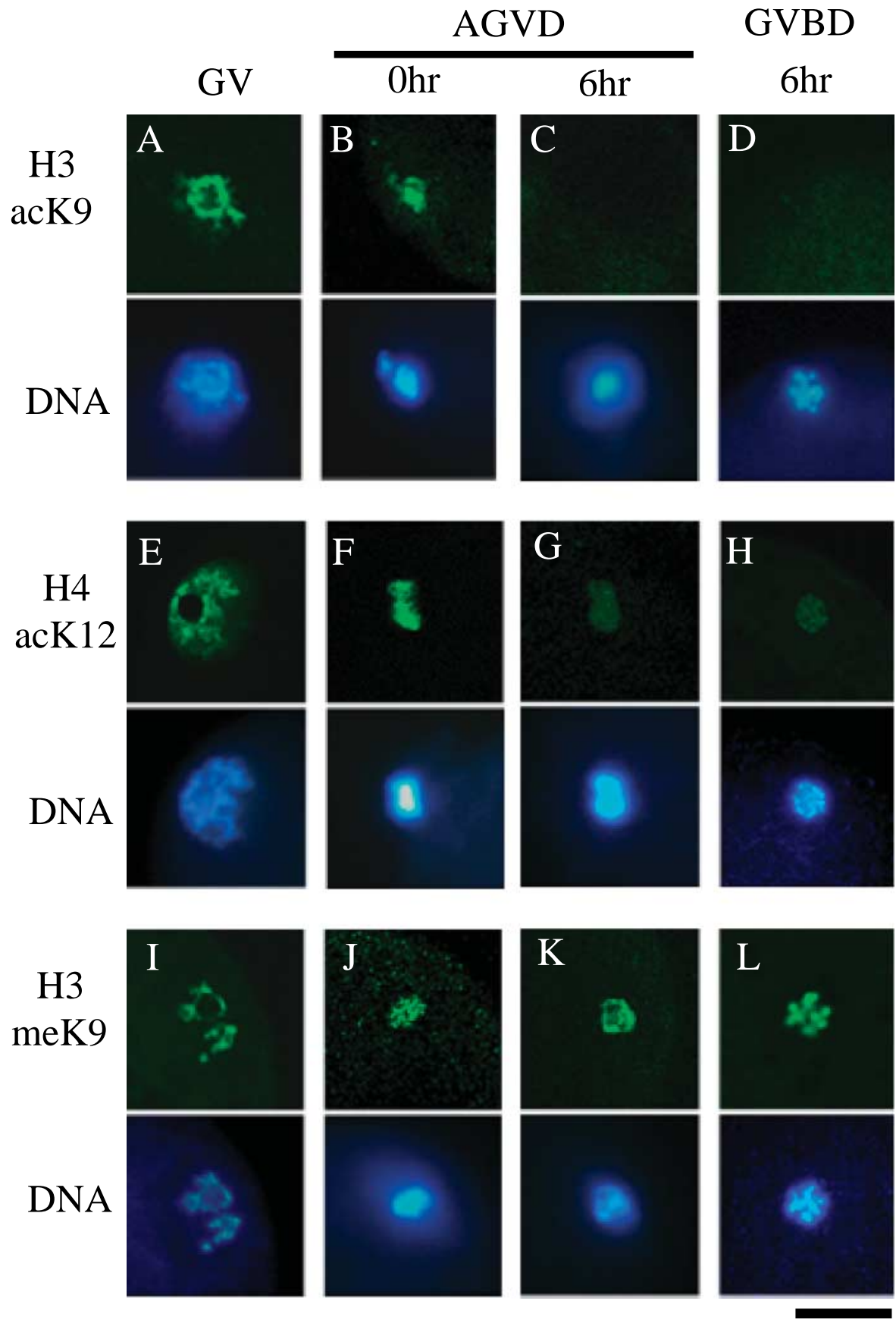

Figure 3 Changes of specific histone modifications in AGVD oocytes. Intact noncultured oocytes at the GV stage (A, $E$ and I) and AGVD oocytes cultured for 0 (B, E and $\mathrm{H}$ ) or $6 \mathrm{~h}(\mathrm{C}, \mathrm{F}$ and $\mathrm{I})$ were immunostained with antibodies specific for lysine 9-acetylated histone $\mathrm{H} 3$ (H3acK9) (A-C), lysine 12-acetylated histone H4 (H4acK12) (E-G), or lysine 9-tri-methylated histone $\mathrm{H} 3$ ( $\mathrm{H} 3$ meK9) (I-K). Typical examples are shown with the first metaphase oocytes at $6 \mathrm{~h}$ after spontaneous GVBD (D, H and L respectively). Each sample was counterstained with Hoechst 33342 to visualize the DNA (lower panels). Experiments were repeated at least three times, and more than 30 oocytes were examined in each experimental group. At least $90 \%$ of examined oocytes in each experimental group showed the same signal strength. Scale bar: $30 \mu \mathrm{m}$. 
When these AGVD oocytes were cultured for $6 \mathrm{~h}$, the histone acetylations in both lysines were remarkably decreased, the signal of $\mathrm{K} 9$ on $\mathrm{H} 3$ becoming undetectable (Fig. 3C) and that of $\mathrm{K} 12$ on $\mathrm{H} 4$ becoming very weak (Fig. 3G). These acetylation levels at $6 \mathrm{~h}$ after AGVD were comparable with those of the first meiosis (MI) oocytes observed at $6 \mathrm{~h}$ after spontaneous GVBD (Fig. 3D and $\mathrm{H}$ ). In contrast, the tri-methylation of $\mathrm{K} 9$ on $\mathrm{H} 3$ was unchanged by the 6-h culturing after AGVD: its signal remained very high (Fig. 3K) like that of $\mathrm{Ml}$ oocytes (Fig. 3L).

\section{Cyclin B/CDK1 and MAPK states in AGVD oocytes}

As MPF and MAPK act ivities increased dramatically just after GVBD in normal porcine oocytes (Naito \& Toyoda 1991, Inoue et al. 1995), we wondered whether the AGVD manipulation induced premature activation of these kinases artificially. Therefore, we next examined the states of MPF subunits, cyclin B and CDK1, and MAPK in 6 h-cultured AGVD oocytes. In intact oocytes, both cyclins B1 and B2, which are necessary for the MPF activation, were undetectable in noncultured and $6 \mathrm{~h}$-cultured oocytes, but were heavily accumulated in 48 h-cultured mature oocytes (Fig. 4A), as reported previously (Naito et al. 1995, Kuroda et al. 2004). In AGVD oocytes, no

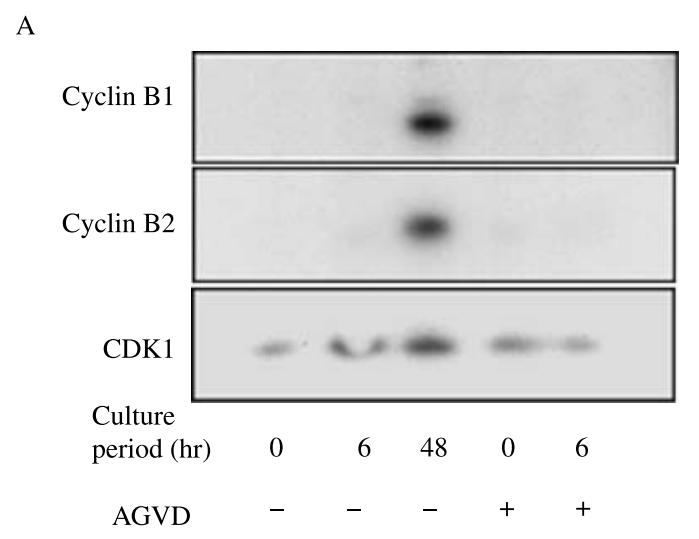

B

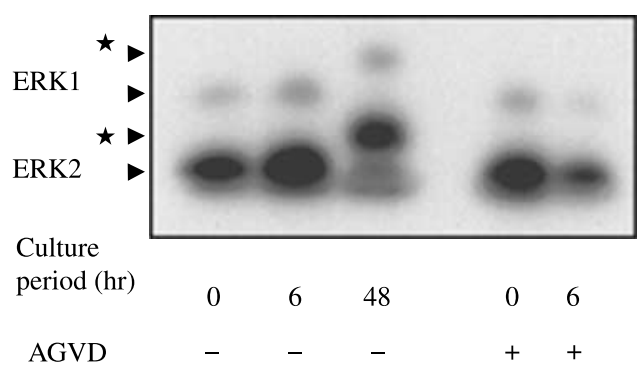

Figure 4 Cyclin B/CDK1 and MAP kinase states in AGVD oocytes. Ten intact or AGVD oocytes were subjected to immunoblotting at the indicated culture periods. (A) Protein levels of cyclin B1 (upper panel), cyclin B2 (middle panel) and CDK1 (lower panel). Different parts of the same membrane were used for detection of each protein. (B) The phosphorylation status of ERK1/2. The phosphorylated and activated bands are indicated by asterisks. cyclin B accumulation was detected at 6-h culturing just as in intact oocytes, indicating that the mixing of GV materials and cytoplasm had no accelerating effect on the cyclin B synthesis (Fig. 4A). The phosphorylation states of ERK1 and ERK2, major MAPKs in porcine oocytes, are shown in Fig. 4B. The shift up bands of ERK1/2, which indicate the phosphorylated and activated forms, were not detected in noncultured $(0 \mathrm{~h})$ or $6 \mathrm{~h}$-cultured oocytes in either the intact or the AGVD group, whereas clear upward shifts in the bands were observed in 48-h-cultured intact oocytes. These results revealed that MPF and MAPK were not activated during the 6-h culture, even in AGVD oocytes (see also Fig. 6).

\section{Effects of roscovitine, cycloheximide and tricostatine $A$ on histone acetylation and kinase activities in AGVD oocytes}

To confirm the dispensability of MPF and MAPK activities for the global deacetylation, we next added roscovitine (Ros), a specific CDK1 inhibitor; cycloheximide (CHX), an inhibitor of protein synthesis, which was necessary for the activation of MPF and MAPK in porcine oocytes (Kalous et al. 1993, Inoue et al. 1996); or tricostatine A (TSA), an inhibitor of HDACs, to the culture medium, and the states of histone acetylation in 6-h-cultured AGVD oocytes were compared with those in noncultured $(0 \mathrm{~h})$ oocytes. As shown in Fig. 5, Ros and $\mathrm{CHX}$ had no effect on the deacetylation of $\mathrm{K} 9$ on $\mathrm{H} 3$ and $\mathrm{K} 12$ on $\mathrm{H} 4$ in AGVD oocytes. In contrast, TSA maintained the histone acetylations unchanged from noncultured oocytes just after manipulation. These results confirm the dispensability of MPF and MAPK activities and also the protein synthesis for histone deacetylations, and suggest the dependence of this deacetylation on HDAC activities.

The activities of MPF and MAPK in these oocytes were assayed, and the results are shown in Fig. 6. The activities of both kinases in the oocytes cultured for $6 \mathrm{~h}$ with or without Ros or $\mathrm{CHX}$ were as low as those in noncultured oocytes, although the MPF and MAPK activities were increased moderately and markedly in 24-h- and 48-hcultured intact oocytes respectively.

\section{Localization of HDAC1 during in vitro maturation of intact and AGVD oocytes}

As the removal of GV membrane was sufficient for the histone deacetylation, the localization of HDAC1 was examined by immunostaining throughout the maturation of intact oocytes, and typical results are shown in Fig. 7A. The porcine HDAC1 was present within the GV in the noncultured GV-stage oocytes, and then was located on the PMI chromosomes after GVBD. The signal strength was relatively weak until the PMI stage. Thereafter, the strong signal was localized on the metaphase chromosomes in the MI and MIl oocytes. In the AGVD oocytes, the HDAC1 localized on the aggregated chromatin just after manipulation, and the appearance of the signal was 


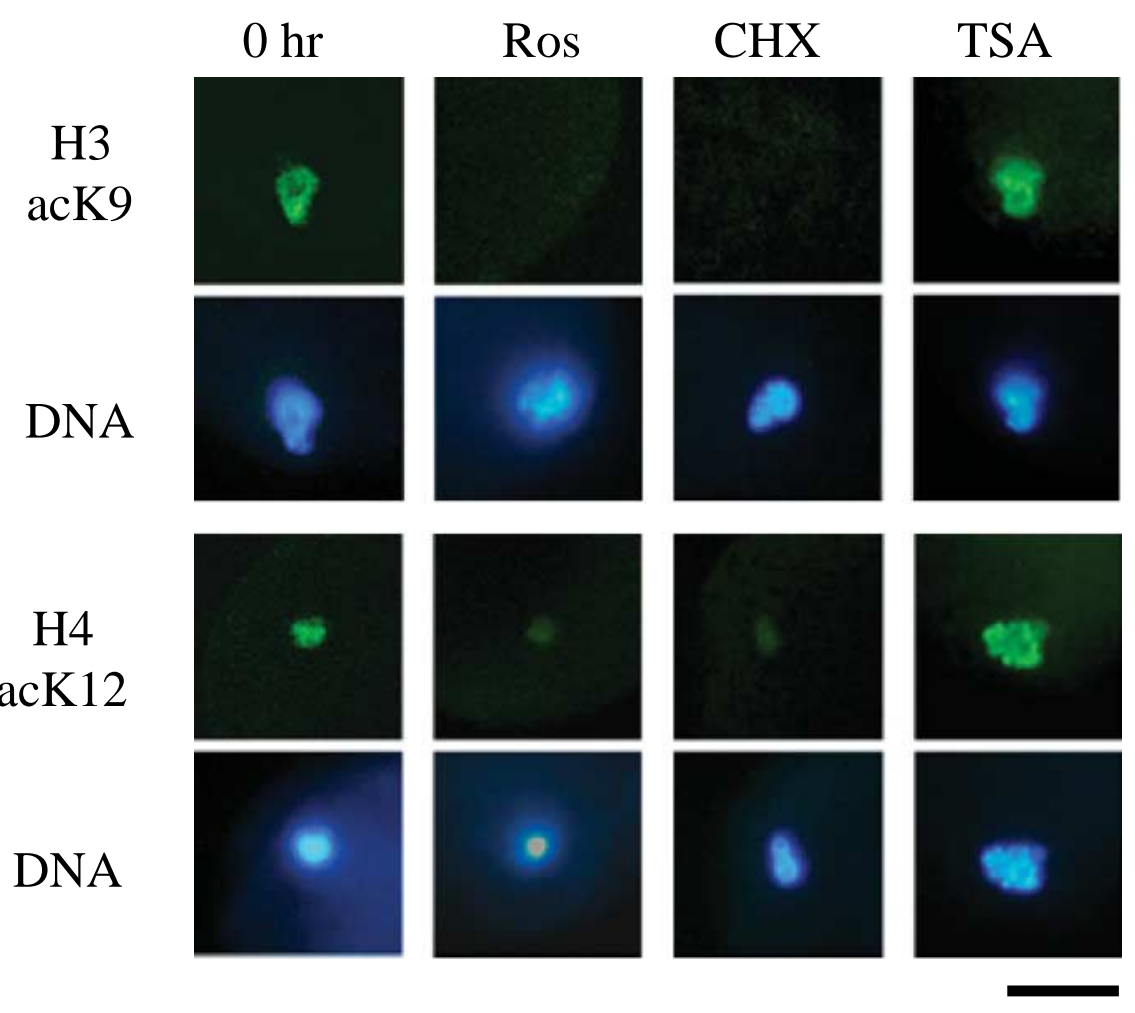

Figure 5 Effects of roscovitine, cycloheximide or tricostatine $A$ on histone acetylation in AGVD oocytes. AGVD oocytes were cultured for $6 \mathrm{~h}$ in the medium containing roscovitine (Ros: $50 \mu \mathrm{M})$, cycloheximide (CHX: $35 \mu \mathrm{M}$ ) or tricostatine $\mathrm{A}$ (TSA: $500 \mu \mathrm{M}$ ), and immunostained with antibodies for lysine 9acetylated histone $\mathrm{H} 3$ (H3acK9) and lysine 12-acetylated histone $\mathrm{H} 4$ (H4acK12). Typical examples are shown with the oocyte just after manipulation and without cultivation $(0 \mathrm{~h})$. Each sample was counterstained with Hoechst 33342 to visualize the DNA (lower panels). Experiments were repeated two times, and more than 20 oocytes were examined in each experimental group. At least $90 \%$ of examined oocytes in each experimental group showed the same signal strength. Scale bar: $30 \mu \mathrm{m}$. very similar to that of the PMI oocytes (Fig. 7B). The signal strength and localization were maintained unchanged in the 6-h-cultured AGVD oocytes.

\section{Discussion}

In the present study, we aimed to determine whether the activities of meiosis-related kinases were required, or the breakdown of GV membrane was sufficient, for the global

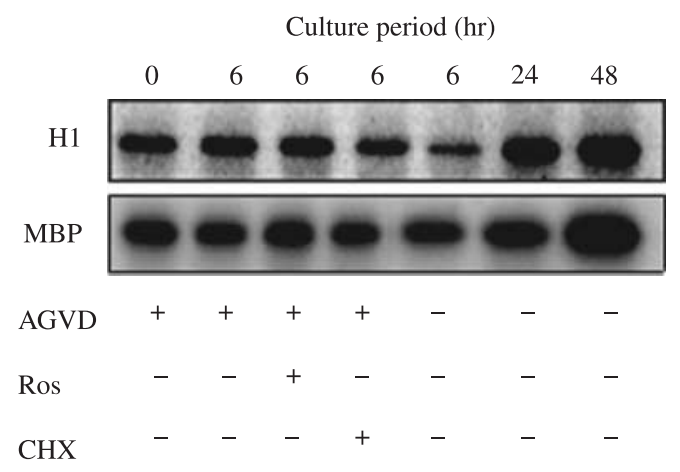

Figure 6 MPF and MAP kinase activities in AGVD oocytes. Intact and AGVD oocytes were cultured for the indicated periods in media with or without roscovitine $(50 \mu \mathrm{M})$ or cycloheximide $(35 \mu \mathrm{M})$. Ten of either type of oocytes were subjected to the kinase assays. The bands of phosphorylated histone $\mathrm{H} 1$, which indicates the MPF activity (upper panel), and the bands of phosphorylated MBP, which indicates the MAP kinase activity (lower panel), were visualized by autoradiography. The experiments were repeated twice with nearly identical results. histone deacetylation that was previously observed after GVBD in the meiosis of mouse and porcine oocytes. (Kim et al. 2003, Akiyama et al. 2004, Endo et al. 2005). To achieve this objective, we destroyed the GV membrane of porcine immature oocytes artificially (artificial GV destruction: AGVD) and found that the signal strength of acetylated histone immunostaining was dramatically decreased $6 \mathrm{~h}$ after the AGVD manipulation. This decrease in immunostaining signals was not attributed to the degenerative changes of oocytes, such as the destruction of nucleosome structure or degradation of histone tails, for the following reasons. Firstly, no degenerative changes were observed morphologically in the AGVD oocytes at this time point ( $6 \mathrm{~h}$ after manipulation), and at least some of the oocytes were able to complete maturation. Second, trimethylation of $\mathrm{K} 9$ in $\mathrm{H} 3$ remained unchanged. Third, the degrees of immunostaining at $6 \mathrm{~h}$ after the AGVD were very similar to those at $6 \mathrm{~h}$ after the spontaneous GVBD reported previously (Endo et al. 2005). Finally, this decrease was completely inhibited by treatment with TSA, an inhibitor of HDACs. These facts indicate that the decrease of immunostaining signals $6 \mathrm{~h}$ after the AGVD manipulation was clearly attributable to the deacetylation of histones by the HDAC activity.

In the present study, the lack of accumulation of cyclin $B$, a regulation subunit of MPF activity, and the lack of MAPK phosphorylation, which indicates MAPK activation, were detected in either the 6-h-cultured AGVD oocytes or the 6-h-cultured intact oocytes, whereas the 48-h-cultured mature oocytes showed marked cyclin B accumulation 
A

HDAC1

DNA
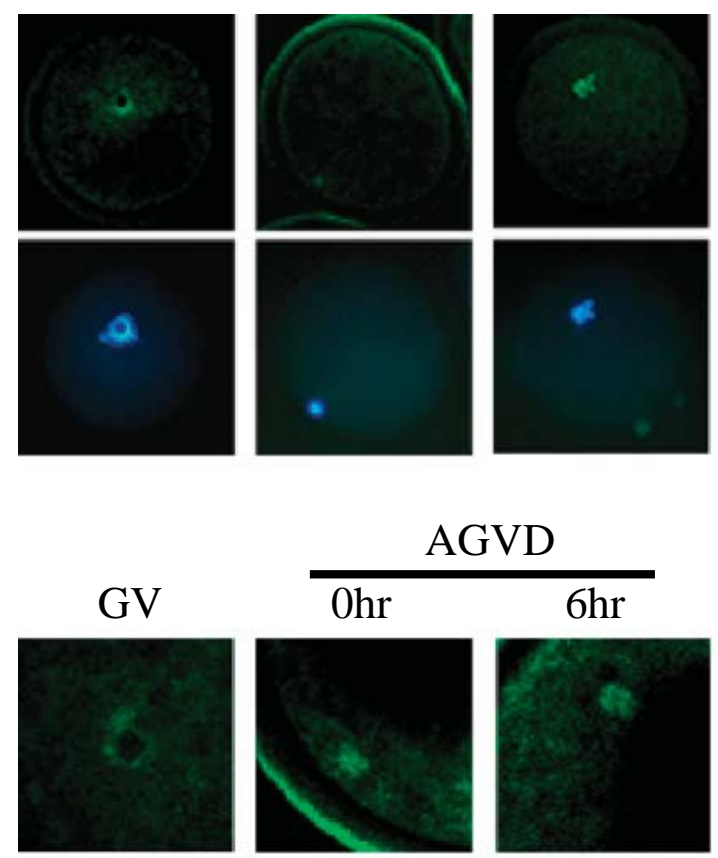

AGVD

B
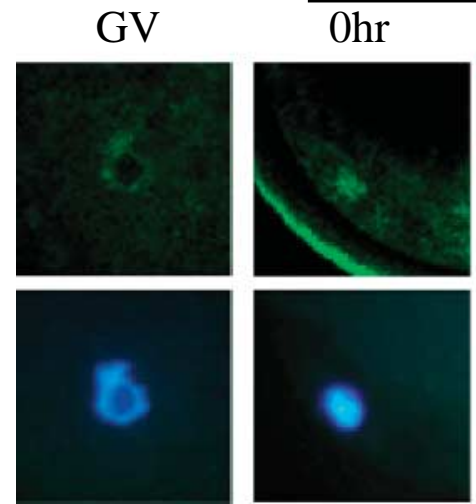

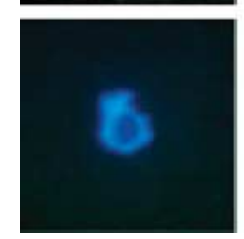

PM I

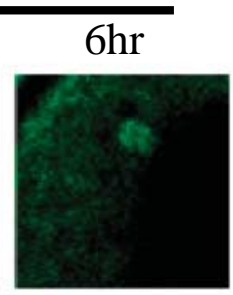

DNA

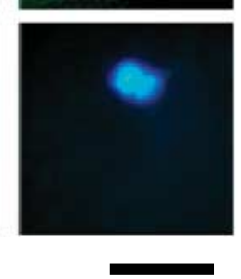

M II
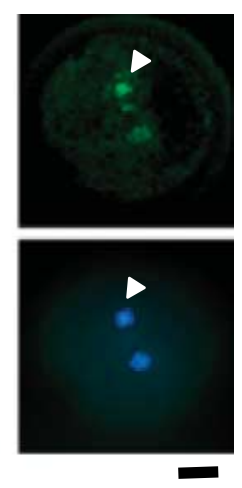

Figure 7 Localization of HDAC1 during in vitro maturation of intact and AGVD oocytes. Porcine oocytes were immunostained with an antibody specific for HDAC1. (A) Intact oocytes were cultured for 0, 24, 30 and $48 \mathrm{~h}$, and typical oocytes at the stages of GV, the first prometaphase (PMI), the first metaphase (MI) and the second metaphase (MII) are shown respectively. Arrowheads in the MII oocytes indicate the first polar bodies. (B) AGVD oocytes cultured for 0 and $6 \mathrm{~h}$ are shown with intact, noncultured oocytes (GV). Each sample was counterstained with Hoechst 33342 to visualize the DNA (lower panels). Experiments were repeated at least three times, and more than 30 oocytes were examined in each experimental group. At least $90 \%$ of stained oocytes in each experimental group showed the same staining pattern. Scale bar: $30 \mu \mathrm{m}$. and MAPK phosphorylation, as reported previously (Inoue et al. 1995, Naito et al. 1995, Kuroda \& Naito 2003). This result suggests that the mixing of GV contents and oocyte cytoplasm by the destruction of the GV membrane did not activate MPF and MAPK after the 6-h culture. It has been reported, however, that immature porcine oocytes contained a negligible amount of cyclin B2, but no cyclin B1, as a form of phosphorylated and inactivated pre-MPF, and it could activate MPF slightly even in the absence of cyclin B synthesis and accumulation (Kuroda et al. 2004). Therefore, we further examined the effects of roscovitine, a potent inhibitor of MPF activity, and $\mathrm{CHX}$, an inhibitor of protein synthesis, on the histone deacetylation of 6-hcultured AGVD oocytes. The results revealed that histone deacetylation occurred after $6 \mathrm{~h}$ of AGVD manipulation even in the roscovitine- or CHX-treated oocytes, which maintained low MPF and MAPK activities comparable with those in noncultured immature oocytes (Naito \& Toyoda 1991, Inoue et al. 1995). These results suggest that the histone deacetylation observed after GVBD during meiotic maturation in porcine oocytes depends only on the breakdown of the GV membrane, and not on the MPF and MAPK activities and protein synthesis. The importance of GVBD for histone deacetylation is supported by a study in which the reprogramming of somatic nuclei injected into the GV of Xenopus oocytes was observed after the rupture of GV membrane (Byrne et al. 2003).

In mouse oocytes, the involvement of MPF activity in histone deacetylation during meiotic maturation has been suggested by experiments in which roscovitine treatment prevented the histone deacetylation after GVBD and induced reacetylation of deacetylated histones (Akiyama et al. 2004). This result, however, does not conflict with the present results, because the roscovitine treatment during the first meiosis inhibited GVBD, and its treatment during the second meiosis induced the escape from the M-phase and subsequent formation of the nuclear membrane. On the other hand, although GVBD occurred in the protein synthesis-inhibited mouse oocytes, the histone deacetylation was also prevented in these oocytes (Akiyama et al. 2004). The cause of this discrepancy is unknown, but the most probable explanation might be the large difference in protein synthesis-dependency during meiotic maturation between mouse and porcine oocytes (Fulka et al. 1986).

The intracellular localization of HDAC1 in porcine oocytes was examined throughout meiosis in the present study, and it was revealed that HDAC1 was present in the GV and localized on the chromosomes after GVBD. When the GV membrane was broken by AGVD, HDAC1 
localized on the clustered chromosomes as in the prometaphase stage after spontaneous GVBD, supporting the notion that the deacetylation after AGVD depends on the physiologic HDAC activity. The presence of mouse HDAC1 and Xenopus HDACm, a homolog of HDAC1, in the GV has been also reported (Ladomery et al. 1997, Ryan et al. 1999, Kim et al. 2003). These HDACs, however, should have been inactive or unable to bind to the nucleosome histones in the GV, because the histones were highly acetylated and were not deacetylated until GVBD. It has been reported that HDAC1 is activated by phosphorylation (Pflum et al. 2001, Tsai \& Seto 2002, Galasinski et al. 2002), and that HDAC does not work as a single enzyme but rather as a complex with multiple components (Brehm et al. 1998, Nan et al. 1998, Tong et al. 1998). Therefore, some cytoplasmic kinases other than MPF and MAPK might phosphorylate and activate HDAC1, or some components in the HDAC complex might be present in cytoplasm. Although we examined only HDAC1 in the present study, the presence of other HDACs has also been reported in mammalian oocytes. Proteins of HDACs 1, 2 and 3 and mRNA of HDAC7 were present in bovine oocytes throughout the maturation period (Segev et al. 2001, McGraw et al. 2003). In mouse oocytes, HDAC6 was present in the cytoplasm during maturation (Verdel et al. 2003). There is yet another possibility, namely, that these HDACs were also present in porcine oocytes and functioned in the cytoplasmic histone deacetylation.

Previously, we reported that the histones deacetylated in the first metaphase were reacetylated at the first anaphase and telophase, and then deacetylated again at the second metaphase, and suggested that histone acetylation during oocyte maturation was dependent on the cell cycle (Endo et al. 2005). The same result has also been reported in mouse oocytes (Akiyama et al. 2004). Because the nuclear membrane is not formed during the transition period from the first meiosis to the second meiosis, the question of how the histone reacetylation is regulated during this period is an intriguing one. It is well accepted that the anaphase-promoting complex (APC), a key ubiquitine ligase during M-phase, is transiently activated during the late metaphase and the anaphase, and ubiquitinates securin and cyclin B for their destruction (Peters 1998, 1999). Since the APC is inactive during the first meiosis until the transient activation at the meiotic transition period followed by abrupt reinactivation during the second meiosis, the APC is a strong candidate for the inactivation of HDACs and induction of histone reacetylation during the meiotic transition period. Hence, further studies, such as an assay of HDAC activities and HDAC protein levels, are clearly required to clarify the regulation mechanism of histone acetylations during oocyte meiosis.

\section{Acknowledgement}

This research was supported by a grant in aid for scientific research (no. 17380173 to $\mathrm{K} \mathrm{N}$ and no. 16380197 to $\mathrm{H} \mathrm{T}$ ) from the Ministry of Education, Science, Sports and Culture of Japan. The authors declare that there is no conflict of interest that would prejudice the impartiality of this scientific work.

\section{References}

Agalioti T, Chen G \& Thanos D 2002 Deciphering the transcriptional histone acetylation code for a human gene. Cell 111 381-392.

Akiyama T, Kim JM, Nagata M \& Aoki F 2004 Regulation of histone acetylation during meiotic maturation in mouse oocytes. Molecular Reproduction and Development $69222-227$.

Brehm A, Miska EA, McCance DJ, Reid JL, Bannister AJ \& Kouzarides T 1998 Retinoblastoma protein recruits histone deacetylase to repress transcription. Nature 391 597-601.

Byrne JA, Simonsson S, Western PS \& Gurdon JB 2003 Nuclei of adult mammalian somatic cells are directly reprogrammed to oct-4 stem cell gene expression by amphibian oocytes. Current Biology $131206-1213$.

Endo T, Naito K, Aoki F, Kume S \& Tojo H 2005 Changes in histone modifications during in vitro maturation of porcine oocytes. Molecular Reproduction and Development 71 123-128 (erratum 398).

Fulka J Jr, Motlik J, Fulka J \& Jilek F 1986 Effect of cycloheximide on nuclear maturation of pig and mouse oocytes. Journal of Reproduction and Fertility 77 281-285.

Galasinski SC, Resing KA, Goodrich JA \& Ahn NG 2002 Phosphatase inhibition leads to histone deacetylases 1 and 2 phosphorylation and disruption of corepressor interactions. Journal of Biological Chemistry 277 19618-19626.

Inoue M, Naito K, Aoki F, Toyoda Y \& Sato E 1995 Activation of mitogen-activated protein kinase during meiotic maturation in porcine oocytes. Zygote 3 265-271.

Inoue M, Naito K, Nakayama T \& Sato E 1996 Mitogen-activated protein kinase activity and microtubule organization are altered by protein synthesis inhibition in maturing porcine oocytes. Zygote $\mathbf{4}$ 191-198.

Kalous J, Kubelka M, Rimkevicova Z, Guerrier P \& Motlik J 1993 Okadaic acid accelerates germinal vesicle breakdown and overcomes cycloheximide- and 6-dimethylaminopurine block in cattle and pig oocytes. Developmental Biology 157 448-454.

Kanno T, Kanno Y, Siegel RM, Jang MK, Lenardo MJ \& Ozato K 2004 Selective recognition of acetylated histones by bromodomain proteins visualized in living cells. Molecular Cell 13 33-43.

Kim JM, Liu H, Tazaki M, Nagata M \& Aoki F 2003 Changes in histone acetylation during mouse oocyte meiosis. Journal of Cell Biology 162 37-46.

Kruhlak MJ, Hendzel MJ, Fischle W, Bertos NR, Hameed S, Yang XJ, Verdin E \& Bazett-Jones DP 2001 Regulation of global acetylation in mitosis through loss of histone acetyltransferases and deacetylases from chromatin. Journal of Biological Chemistry 276 38307-38319.

Kuroda T \& Naito K 2003 Localization and function of cyclin B1 and cyclin B2 during porcine oocyte maturation. Journal of Mammalian Ova Research 20 93-98.

Kuroda T, Naito K, Sugiura K, Yamashita M, Takakura I \& Tojo H 2004 Analysis of the roles of cyclin B1 and cyclin B2 in porcine oocyte maturation by inhibiting synthesis with antisense RNA injection. Biology of Reproduction 70 154-159.

Ladomery M, Lyons S \& Sommerville J 1997 Xenopus HDm, a maternally expressed histone deacetylase, belongs to an ancient family of acetyl-metabolizing enzymes. Gene 198 275-280.

Laemmli UK 1970 Cleavage of structural proteins during the assembly of the head of bacteriophage T4. Nature 227 680-685. 
McGraw S, Robert C, Massicotte L \& Sirard MA 2003 Quantification of histone acetyltransferase and histone deacetylase transcripts during early bovine embryo development. Biology of Reproduction $68383-389$.

Naito K \& Toyoda Y 1991 Fluctuation of histone H1 kinase activity during meiotic maturation in porcine oocytes. Journal of Reproduction and Fertility 93 467-473.

Naito K, Fukuda Y \& Toyoda Y 1988 Effects of porcine follicular fluid on male pronucleus formation in porcine oocytes matured in vitro. Gamete Research 21 289-295.

Naito K, Hawkins C, Yamashita M, Nagahama Y, Aoki F, Kohmoto K, Toyoda Y \& Moor RM 1995 Association of p34 ${ }^{\text {cdc2 }}$ and cyclin B1 during meiotic maturation in porcine oocytes. Developmental Biology 168 627-634.

Naito K, Kagii H, Iwamori N, Sugiura K, Yamanouchi K \& Toji H 1999 Establishment of a small-scale Western blotting system named as 'micro-Western blotting' for mammalian ova analysis. Journal of Mammalian Ova Research 16 154-157.

Nan X, Ng HH, Johnson CA, Laherty CD, Turner BM, Eisenman RN \& Bird A 1998 Transcriptional repression by the methyl-CpG-binding protein MeCP2 involves a histone deacetylase complex. Nature 393 386-389.

O'Neill LP \& Turner BM 1995 Histone H4 acetylation distinguishes coding regions of the human genome from heterochromatin in a differentiation-dependent nut transcription-independent manner. EMBO J 14 3046-3957.

Peters JM 1998 SCF and APC: the Yin and Yang of cell cycle regulated proteolysis. Current Opinion in Cell Biology 10 759-768.

Peters JM 1999 Subunits and substrates of the anaphase-promoting complex. Experimental Cell Research 248 339-349.

Pflum MK, Tong JK, Lane WS \& Schreiber SL 2001 Histone deacetylase 1 phosphorylation promotes enzymatic activity and complex formation. Journal of Biological Chemistry 276 47733-47741.

Ryan J, Llinas AJ, White DA, Turner BM \& Sommerville J 1999 Maternal histone deacetylase is accumulated in the nuclei of Xenopus oocytes as protein complexes with potential enzyme activity. Journal of Cell Science 112 2441-2452.

Sarmento OF, Digilio LC, Wang Y, Perlin J, Herr JC, Allis CD \& Coonrod SA 2004 Dynamic alterations of specific histone modifications during early murine development. Journal of Cell Science 117 4449-4459.

Segev H, Memili E \& First NL 2001 Expression patterns of histone deacetylases in bovine oocytes and early embryos, and the effect of their inhibition on embryo development. Zygote 9 123-133.

Spencer VA \& Davie JR 1999 Role of covalent modifications of histones in regulating gene expression. Gene 240 1-12.

Strahl BD \& Allis CD 2000 The language of covalent histone modifications. Nature 403 41-45.

Sugiura K, Naito K, Iwamori N, Kagii H, Yamashita M, Goto S, Ohashi S, Yamanouchi K \& Tojo H 2001 Studies on the germinal vesicle requirement for MAP kinase activities in porcine oocyte maturation. Molecular Reproduction and Development $\mathbf{5 9}$ $215-220$.

Tong JK, Hassig CA, Schnitzler GR, Kingston RE \& Schreiber SL 1998 Chromatin deacetylation by an ATP-dependent nucleosome remodelling complex. Nature 395 917-921.

Toyoda Y, Yokoyama M \& Hosi T 1971 Studies on the fertilization of mouse eggs in vitro. In vitro fertilization of eggs by fresh epididymal sperm. Japanese Journal of Animal Reproduction 16 147-151.

Tsai SC \& Seto E 2002 Regulation of histone deacetylase 2 by protein kinase CK2. Journal of Biological Chemistry 277 31826-31833.

Turner BM 2002 Cellular memory and the histone code. Cell $\mathbf{1 1 1}$ 285-291.

Verdel A, Seigneurin-Berny D, Faure AK, Eddahbi M, Khochbin S \& Nonchev S 2003 HDAC6-induced premature chromatin compaction in mouse oocytes and fertilised eggs. Zygote 11 323-328.

Wu J \& Grunstein M 200025 years after the nucleosome mode: chromatin modifications. Trends in Biochemical Sciences 25 619-623.

Received 11 August 2005

First decision 13 September 2005

Revised manuscript received 27 September 2005

Accepted 22 November 2005 